\title{
Self-adaptive Prototype for Seat Adaption
}

\author{
Gian Mario Bertolotti, Andrea Cristiani, \\ Remo Lombardi \\ Dip. Informatica e Sistemistica \\ Università di Pavia \\ Pavia, Italy \\ e-mail: gianmario.bertolotti@unipv.it
}

\author{
Marko Ribarić, Nikola Tomašević, \\ Mladen Stanojević \\ Institute Mihajlo Pupin \\ University of Belgrade \\ Belgrade, Serbia \\ e-mail: mladen.stanojevic@pupin.rs
}

\begin{abstract}
Self-adaptive prototype for seat adaptation aims at enhancing the physical comfort of a driver by taking into account not only the state of the environment (state of the road, car settings), but also the driver's emotional, cognitive and physical state. To implement this prototype we used a REFLECTive middleware, which provides a programming framework for the development of pervasive-adaptive applications. The REFLECTive middleware supports selfadaptive behavior and is generally composed of three tiers: Tangible tier contains services that read sensors data and send commands to actuators; REFLECTive tier is responsible for analyzing the data collected from sensors and for defining the actions that will be performed by actuators; Application tier facilitates high-level decision making. The seat adaptation prototype uses the information about Center of Pressure (COP) speed and number of bumps to determine the driver's physical state, and then it combines this information with the driver's cognitive and emotional state to figure out if the driver feels uncomfortable, and to change the state of seat cushions in an attempt to make driver feel more comfortable. The components of the seat adaptation prototype in the REFLECTive and Application tier are implemented using reaction rules.
\end{abstract}

Keywords-pervasive adaptation; middleware; driving experience; physical comfort; reaction rules

\section{INTRODUCTION}

One of the main objectives of the REFLECT project, which is funded by the EU Seventh Framework Programme, ICT Thematic Priority, Challenge 8: Future and Emerging Technologies, Objective ICT-2007.8.2: Pervasive adaptation, is to support the development of pervasive adaptive systems. The seat adaptation prototype, which is currently being developed within the project, has a focus on identifying and possibly enhancing the physical state (comfort) of end users (drivers) in an attempt to verify the applicability, usefulness and self-adaptive capabilities of the proposed REFLECTive middleware.

Seamless and implicit human-computer interaction is an important characteristic of smart technology [1]. In order for technology to be "smart", the system must have some means of assessing the system environment and user state. The development of smart computer systems calls for technology to interface with user behavior in order to adapt appropriately and in real-time.

"What you like is what you get" [2] is the ultimate principle of this new generation of adaptive pervasive technology. Each interaction has unique characteristics that may be determined by the person, the system, or the environment. The purpose of dialogue design is to create an interface to maximize performance efficiency or safety, which represents a tacit attempt to "standardize" the dynamics of the interaction. Similarly, human factors and ergonomics research has focused on the optimization of interaction for a generic "everyman" user. Adaptive and personalized computing represents a challenge to the twin concepts of a standard interaction or a standard user. These systems incorporate an improvisatory element where user and system respond to feedback from each other in realtime. Also, interactions with such systems may be tailored to the specific needs of an individual in a defined place at a precise time. This shift from designing for the general to the specific attributes of the user has been called individuation [3], which is "directed to explore ways through which each and every individual can customize his or her tools to optimize the pleasure and efficiency of his or her personal interaction". Individualization requires proactive intelligent adaptation of the system to tailor the interaction to the individual. This level of adaptation is achieved by monitoring the user in order to learn needs and preferences and predict intentions in real-time. This process has been called a bio-cybernetic loop [3,4], where a technical system influences behavior via an iterative process according to the bio-behavioral response from the user.

The deployment of a bio-cybernetic loop in a real-time interactive design will dramatically change the landscape of human-computer interaction. Equipped with numerous sensor devices that observe users in specific task environments, smart systems are able to derive users' emotional [5], cognitive [6] and physical states and to adapt its functioning accordingly.

The dynamics of the reflective computing system is based upon the bio-cybernetic loop as described originally in [7]. This loop describes how psycho-physiological data regarding the status of the user is captured, analyzed and converted to a computer control input in real-time. The 
function of the loop is to monitor changes in user state in order to initiate an appropriate adaptive response.

The aim of the seat adaptation prototype is to monitor the postural behaviour of a car driver, detecting whether he/she feels uncomfortable in the seat, and - if this is the case - to act on the car seat in order to help the driver in finding a more comfortable position.

This prototype is based on the REFLECTive middleware [8], [9] proposed and developed within the REFLECT project. The major goal of the REFLECTive middleware is to provide a common approach for the design, development and deployment of the pervasive adaptive systems, i.e. the systems that are sensitive to emotional cognitive and physical states of the users involved in a particular task or situation.

The REFLECTive middleware is a result of the systematic engineering approach, which insures that the key requirements of adaptivity, reusability and flexibility are fulfilled. The pervasive adaptive systems developed using the REFLECTive middleware seamlessly integrate in the environment of their user, and make use of available physical devices to sense and derive the state of the environment and user, detect the user's current state, and finally try to enhance the efficiency of a user in performing his/her task by changing the conditions of the environment to allow a user to perform the current tasks more efficiently. These systems hence use sensors to provide them with information about the environment and users, and actuators to influence them.

The seat adaptation prototype uses the Centre of Pressure (COP) speed to identify the physical state of a driver and the number of bumps as an indicator of the state of road. This information is then combined with the car settings and driver's cognitive and emotional state to determine whether any action should be performed to enhance the comfort of the driver by inflating/deflating seat cushions. The logic described is implemented using reaction rules [10].

The organization of the paper is the following: Section II describes means for detecting the physical state of the driver and influencing his/her comfort; Section III gives an overview of REFLECTive middleware; Section IV presents the system design; Section V describes the system components used to implement the prototype; Section VI gives the reaction rules used to program the prototype logic; Section VII states the conclusions.

\section{Detecting UnCOMFortable Positions}

As reported by Harrison et al. in [11], sitting posture concepts are subject for discussion among scientists for over a century, the first article dating back to 1884. Most of the works in literature deal with the assessment of sitting comfort and discomfort, and many of them try to give an explanation of the physical causes of discomfort, focusing on prolonged sitting posture situations. Localized contact stresses are identified as one of the main responsible of sitting discomfort: prolonged pressures on the back or lower legs can eventually reduce the blood flow at the buttock/seat interface and in legs and feet [12].

As presented by Hermann in [13], some researchers use the terms "fidgeting" or "in chair movement" to describe the constant slight posture changes that subjects do while sitting. An increasing of these movements has been directly linked to an increasing of discomfort perceived by the sitter. While sitting in a car during driving, the pilot and the passengers are also subjected to vibrations generated by the vehicle and the different types of road surfaces. To compensate for these vibrations, the human body reacts with micro-muscle adjustments. This behavior, combined with the above-mentioned seated-position issues lead to a faster degradation of perceived comfort $[13,14]$.

Although sitting discomfort has been traditionally studied using subjective rating scales, continuous objective measures have been preferred in recent works, because of sitting discomfort's dynamic nature $[15,16]$. To do this, most research groups base their studies on measurements made by means of electronic instrumentation, like optical systems to detect body angles, and pressure sensors systems. Pressure mats are largely used to acquire pressure maps at driver's buttock/seat and driver's back/backseat interfaces. [12,15,17-21].

The Centre Of Pressure (COP) is one of the most used parameter for the study of human posture, both standing (in that case force platforms or insoles are used), and sitting posture $([13,15,18]$,$) . It is defined as "the point of$ application of the result of vertical forces acting on the surface of support" [13]; the latter is the ground in case of standing posture, and the seat in case of sitting posture. To be precise, for car sitting posture more than one surface of support should be considered, as the body of the pilot is in contact with the seat, the backrest, the pedals, and the steering wheel. Nevertheless, results from [17] suggest that driver-seat interaction plays a crucial role in sitting strategies, while other interactions (such as driver-backseat) seem to be less relevant. Of course, in real driving performances, COP modifications (at driver-seat interface) are to be expected when car driving activities are performed. For example, it is reasonable that the use of a pedal results in an increasing of pressure on the area under pilot's tight.

Various experiments [11,15-18,21] have been done, mainly in past ten years, on car seat comfort and discomfort, discussing both subjective information connected to comfort/discomfort perception (by means of questionnaires), and objective measures (e.g. through pressure sensors). Nevertheless, these works focussed only on the assessment or measure of comfort/discomfort. The innovation of the present project is the loop control system, that is to say that we also try to act on the seat. We have done (or planned to do) some experiments aiming at understanding which is/are the most appropriate strategy/ies to adopt for the seat adaptation.

The pressure map at the driver-seat interface is used as an indicator of sitting behaviour. The raw data, collected by 
means of a matrix of pressure sensors and a proper acquisition device, are processed to calculate the Centre Of Pressure (COP), a parameter which is broadly used [12$16,18,20]$ to study human posture.

Given a matrix of $n$ pressure sensors, the COP coordinates (in the XY plane, with respect to a pre-fixed point taken as a reference) at time $t$ are calculated as follows:

$x_{C O P}(t)=\frac{\sum_{i=1}^{n} P_{i}(t) \cdot x_{i}}{\sum_{i=1}^{n} P_{i}(t)} \quad y_{C O P}(t)=\frac{\sum_{i=1}^{n} P_{i}(t) \cdot y_{i}}{\sum_{i=1}^{n} P_{i}(t)}$

Where:

$P_{i}(t)$ is the pressure value detected by the $i^{\text {th }}$ sensor at time t;

$x_{i}$ is the $\mathrm{i}^{\text {th }}$ sensor's horizontal coordinate;

$y_{i}$ is the $\mathrm{i}^{\text {th }}$ sensor's vertical coordinate.

This information and potentially other additional data coming both from the driver (e.g. pressure values from specific areas of the driver-seat interface), and from the car (e.g. speed, acceleration) - are elaborated by the system. The latter tries then to determine if the driver is feeling uncomfortable with the seat; then it decides what action should be taken.

Modern top level car seats are provided with a wide range of adjustments: the driver, before starting the engine, can easily tune the seat position according to his/her preference. Nevertheless, we cannot consider to act on each adjustment when the car is running, due to obvious safety issues. For this reason we decided to act on inflatable air cushions ("barilotti") which are built inside the seat. Connected to these barilotti is an air pump. Acting on the latter and on pneumatic valves trough digital commands inflate or deflate air into/from the formers, resulting in an increasing or decreasing support for the driver's body and then in a modification of his/her postural behaviour.

\section{REFLECTIVE MIDDLEWARE}

The REFLECTive middleware for pervasive adaptive systems has been developed using the software components paradigm and implemented in the Java programming language on top of OSGI environment. Software components are units of software that make their communication capabilities explicit by means of ports (software types that describe the set of messages that can be received or send). The component based approach is highly dynamic, re-configurable and re-usable concept that allows for development of highly generic software. The basic feature of developed pervasive-adaptive software framework is to provide an interface for the four basic operations of configuration for component-based systems: creation and removal of components, and connecting and disconnecting component ports. With the help of these primitives, component based applications can be configured and reconfigured, i.e. assembled during start-up and altered at runtime.

The pervasive adaptive applications are organized in three layers within REFLECTive middleware: the Tangible Layer, the Reflective Layer, and the Application Layer. Tangible Layer deals with interfacing with devices outside the system. For sensors, this means wrapping device drivers to access input data, and wrapping feature extraction code operating on the raw signals. The Reflective Layer constitutes a pool of reusable software components that can be used to analyze the set of features detected by sensors, and adaptive components used to plan the actions that will be performed by actuators. The Application Layer contains all application-specific components and the assembly defining the structure of the application: how services and components are to be configured and connected.

Tangible Layer is the lowest layer of the system, which implements the concept of awareness, interfaces/connects to the sensor and actuator devices and provides the needed functionality (in form of atomic services) to the rest of the system. In the case of collecting multiple psychophysiological features - it provides the Reflective Layer with concrete values that can be used in deducing user's cognitive, emotional and physical states. It also executes commands (coming from the higher levels) needed to control the actuator devices. The Tangible Layer is capable of autonomous processing and can execute urgent (safety) actions reflecting the overall system strategy and performing improvisation as a form of immediate adaptation.

\section{SySTEM DESIGN}

In this section we will describe the application of REFLECTive middleware applied on the Seat Adaptation prototype. The specific architecture used for this use case is presented in Fig. 1.

The Tangible Layer contains devices and the corresponding controllers for the Vehicle CAN Bus, Accelerometer, Seat Mat (sensor) and Seat Cushions (actuators). The sensor data generated by the Accelerometer, Seat Mat and Seat Cushions are collected and processed by the UP Controller. The Accelerometer measures the vertical acceleration of the car and this information is then processed by the UP Controller to count the number of bumps in the given time period, which is the indication of the state of the road. The Seat Mat sensor sends the information to the UP Controller, which calculates the current Centre of Pressure (COP) and current COP Speed and passes it to the Physical Analysis component, while the Seat Adaptation component sends commands to the Seat Adaptation Service and UP controller to implement seat adaptation.

At the REFLECTive Layer we have Cognitive Analysis, Emotional Analysis, Physical Analysis components and Seat Adaptation component. Cognitive Analysis, Emotional Analysis and Physical Analysis components inform the Seat Adaption Manager about the changes of user's cognitive, emotional and physical state, while Seat Adaptation 
component receives the commands from the Seat Adaption Manager when seat adaptation is needed. If seat adaptation is needed, the Seat Adaptation component uses the information about car settings, state of the road, user's physical state and state of seat cushions obtained from the Physical Analysis component to determine which seat adaptation action should be performed.

The Application Layer contains only one component Seat Adaptation Manager, where the logic of the Seat Adaptation use case is implemented.

\section{SySTEM COMPONENTS}

All system components used in Seat Adaptation use case are represented in Fig. 1. At the Tangible Layer we have Car Settings, Bump, COP and Cushions Services, which provide the information needed to determine the physical state of the driver and to perform the required seat adaptation. On the other hand Seat Adaptation Service enables the control of cushions.

The Cognitive and Emotional Analysis components use the sensor readings (pulse, respiration, skin conductance, skin temperature, etc.) to determine the corresponding cognitive and emotional state of the driver, while the Physical Analysis component uses the information about the state of the road and COP speed to determine the driver's physical state.

The Seat Adaptation Manager receives the information about the driver's cognitive, emotional and physical state from the Cognitive Analysis, Emotional Analysis and Physical Analysis components and decides, based on this information, whether the seat should be adapted or not. High COP speed on a flat road may indicate that the driver feels uncomfortable, however it can also be a consequence of driver's emotional state (anger, nervousness, etc.) or high cognitive load. So the Seat Adaptation Manager analyses the driver's cognitive, emotional and physical state and initiates seat adaptation only in situations when the cognitive load of the driver is not high, emotional state is neutral and physical state is recognized as discomfort.

Based on the request from the Seat Adaptation Manager, that the seat should be adapted, Seat Adaptation component sends commands to Seat Adaptation Service to inflate or deflate some cushions based on the information about the COP speed, number of bumps and state of cushions. These commands are finally executed by the UP Controller.

\section{SEAT AdAPTATION REACTION RULES}

Reaction rules (Table 1) used to implement the logic of the seat adaptation prototype are grouped according to their main role into three groups: analysis, management and adaptation. In the analysis groups we have two rules using to detect physical discomfort of the driver. The only rule in the management group is used to detect the situation when seat adaptation is actually required. Finally, the rules from the adaptation group are used to inflate or deflate cushions.

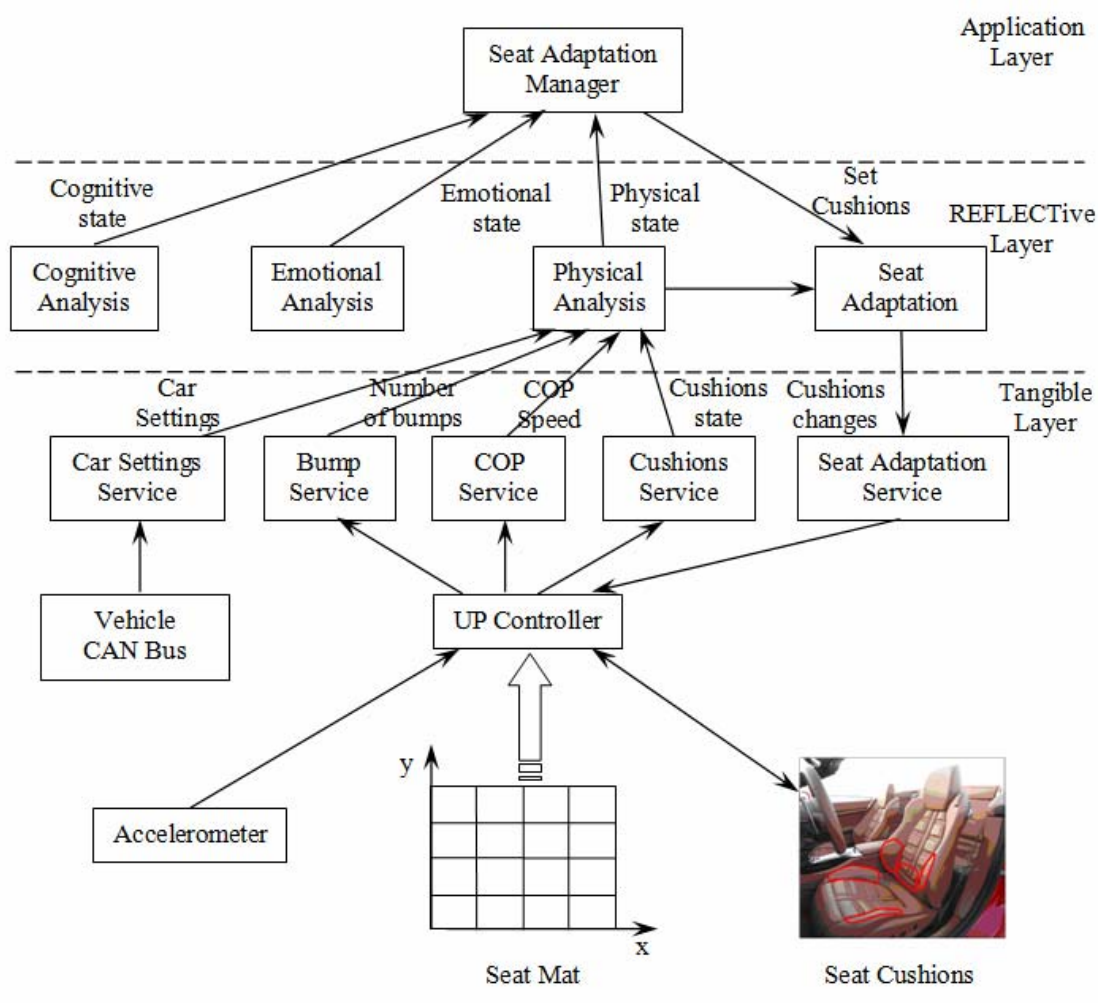

Figure 1. REFLECTive Architecture for the Seat Adaptation use case 
Two time frames are used to provide different seat adaptations depending on the road surface (bumpy, flat): when the number of bumps is high, then COP speed increases and the pilot becomes tired early. Turning on the cushions helps the pilots to compensate vibrations and to feel more comfortable.

If the emotional state of the pilot is not neutral or the cognitive load is high or setting of the car is "sport", then it is not productive to change the state of the seat.

If the road is flat and the driver feels uncomfortable (COP speed is high) we could improve the comfort by changing the state of the cushions.

When time frame is 20 minutes only the last value of COP speed is taken into account.

TABLE I. SEAT AdAPTATION REACTION RULES

\begin{tabular}{|c|c|c|}
\hline No. & & Reaction rules \\
\hline \multicolumn{3}{|r|}{ Physical Analysis } \\
\hline 1. & $\begin{array}{l}\text { IF } \\
\text { THEN }\end{array}$ & $\begin{array}{l}\text { Number of bumps }=\mathrm{HIGH} \\
\text { Physical state }=\text { DISCOMFORT }\end{array}$ \\
\hline 2. & $\begin{array}{l}\text { IF } \\
\text { THEN }\end{array}$ & $\begin{array}{l}\text { Number of bumps = LOW AND } \\
\text { COP speed = HIGH } \\
\text { Physical state = DISCOMFORT }\end{array}$ \\
\hline \multicolumn{3}{|r|}{ Seat Adaptation Manager } \\
\hline 3. & THEN & $\begin{array}{l}\text { Emotional state = NEUTRAL AND } \\
\text { Cognitive load = NOT HIGH AND } \\
\text { Physical state = Discomfort } \\
\text { SET CUSHIONS }\end{array}$ \\
\hline \multicolumn{3}{|r|}{ Seat Adaptation } \\
\hline 4. & $\begin{array}{l}\text { IF } \\
\text { THEN } \\
\text { ELSE }\end{array}$ & $\begin{array}{l}\text { Number of bumps }=\mathrm{HIGH} \\
\text { Time frame }=1 \\
\text { Time frame }=20\end{array}$ \\
\hline
\end{tabular}

\begin{tabular}{|c|ll|}
\hline 5. & IF & $\begin{array}{l}\text { SET CUSHIONS AND } \\
\text { Car setting = COMFORT AND } \\
\text { Number of bumps = HIGH AND } \\
\text { Cushions }=\text { OFF } \\
\text { THEN } \\
\text { Cushions = ON }\end{array}$ \\
\hline 6. & IF & $\begin{array}{l}\text { SET CUSHIONS AND } \\
\text { Car setting = COMFORT AND } \\
\text { Number of bumps = LOW AND } \\
\text { COP speed = HIGH AND }\end{array}$ \\
\hline
\end{tabular}

\begin{tabular}{|l|ll|}
\hline THEN & $\begin{array}{l}\text { Cushions }=\text { OFF AND } \\
\text { Time frame }=20 \text { AND } \\
\text { MOD (time, time frame) }=0 \\
\text { Cushions }=\text { ON }\end{array}$ \\
\hline IF & $\begin{array}{l}\text { SET CUSHIONS AND } \\
\text { Car setting }=\text { COMFORT AND } \\
\text { Number of bumps = LOW AND } \\
\text { COP speed }=\text { HIGH AND } \\
\text { Cushions }=\text { ON AND } \\
\text { Time frame }=20 \text { AND } \\
\text { MOD (time, time frame) }=0 \\
\text { Cushions }=\text { OFF } \\
\text { THEN }\end{array}$ \\
& \\
\hline
\end{tabular}

\section{CONCLUSIONS}

The seat adaptation prototype described within this paper illustrates the use of the REFLECTive middleware in automobile industry with a focus on enhancing the driver's comfort. More precisely, within this prototype the sensors are used to continuously measure the driver's posture, mood and cognitive load and, according to a proper "seat adaptation strategy", actuators integrated in the seat are activated in order to modify the seat's shape (and therefore driver's posture and feeling of comfort). Since top-level cars are provided with a number of actuators integrated in the seat, it is reasonable to try to use one (or a group) of them for the above-described purpose. In order to verify the performance and selfadaptive capabilities of the developed pervasive-adaptive middleware, the seat adaptation prototype will be tested in real driving conditions.

The seat mat at the driver's seat is used as a sensor for driver's moving patterns. The raw data, collected by a matrix of pressure sensors and a proper acquisition device, are processed to calculate the COP coordinates, and then the COP speed. The seat adaptation prototype is based on the COP speed parameter, because it reflects the "fidgeting" of the driver when he/she starts feeling uncomfortable. As it is proven that vibrations play an important role in driver's feeling of comfort, vertical acceleration is another parameter that is important. This information reflecting the state of the road (bumpy/flat) combined with the COP speed can be used to infer the conclusion about the driver's feeling of comfort/discomfort.

The seat adaptation prototype was implemented using a component-based approach of the REFLECTive middleware, where at the Tangible layer we have services that collect information from sensors (car settings, bumps, COP speed, state of cushions) and actuator service that controls seat cushions; at the Reflective layer we have components that perform the analysis of driver's 
cognitive, emotional and physical state and the seat adaptation component; at the Application layer we have only one component - seat adaptation manager, which, based on the information about current driver's state, makes a decision whether seat adaptation is required or not. All components at the Reflective and Application layers are implemented using the reaction rules, which provide the needed intelligent reactivity of the seat adaptation prototype.

Since the prototype has been developed, the next step will be to test it in the real environment and to use the feedback to improve it.

\section{ACKNOWLEDGMENT}

This work has been partly supported by the EU Seventh Framework Programme project REFLECT (Pr. No.: FP7-215893).

\section{REFERENCES}

[1] R., Picard, "Affective Computing", Boston MA, MIT Press, 1997.

[2] Y. Tong, W. Liao, Q. Ji, "Facial action unit recognition by exploiting their dynamics and semantic relationships", IEEE Transactions on Pattern Analysis and Machine Intelligence, Vol. 29, No. 10, 2007, pp. 1683-1699.

[3] A.B. Ashraf et al. "The painful face: pain expression recognition using active appearance models", Proceedings of the ACM International Conference on Multimodal Interfaces (ICMI'07), 2007, pp. 9-14.

[4] J. Wang, L. Yin, X. Wei, Y. Sun, "3D Facial Expression Recognition Based on Primitive Surface Feature Distribution", Proceedings of the 2006 IEEE Computer Society Conference on Computer Vision and Pattern Recognition, Vol. 2, 2006, pp. 1399-1406.

[5] B. Fasel, F. Monay, D. Gatica-Perez, "Latent semantic analysis of facial action codes for automatic facial expression recognition", Proceedings of the 6th ACM SIGMM international workshop on Multimedia information retrieval, 2004, pp.181-188.

[6] M. Yeasin, B. Bullot, R. Sharma, "Recognition of facial expressions and measurement of levels of interest from video", IEEE Transactions On Multimedia, Vol.8, No. 3, 2006, pp. 500-507.

[7] A.T. Pope, E.H. Bogart, D.S. Bartolome, "Biocybernetic system evaluates indices of operator engagement in automated task", Biological Psychology, 40, 1995, pp. 187195.

[8] M. Wirsing, G. Beyer, M. Hammer, C. Kroiss, A. Schroeder, M. Stanojevic, "Requirements and design", Deliverable D2.1 of REFLECT project, 2009. http://reflect.pst.ifi.lmu.de/images/pdf/d2.1.pdf.

[9] A. Schroeder, M. van der Zwaag, M. Hammer, "A Middleware Architecture for Human-Centred Pervasive Adaptive Applications", Second IEEE International Conference on Self-Adaptive and Self-Organizing Systems Workshops, October 2008, Venice, Italy, pp. 138-143.

[10] A. Paschke, A. Kozlenkov, "Rule-based event processing and reaction rules". In RuleML, Lecture Notes in Computer Science Vol. 5858, Springer, 2009, pp. 53-66.

[11] D.D. Harrison, S.O. Harrison, A.C. Croft, D.E. Harrison, and S.J. Troyanovich, "Sitting biomechanics part i: Review of the literature," Journal of Manipulative and Physiological Therapeutics, Vol. 22, No. 9, 1999, pp. 594 609.

[12] H. Miller, "Body support in the office: Sitting, seating, and low back pain", Tech. Rep., Technical report, 2002.

[13] S. Hermann, "Exploring sitting posture and discomfort using nonlinear analysis methods," IEEE Transactions on Information Technology in Biomedicine, Vol. 9, No. 3, 2005, pp. 392-401.

[14] W. El Falou, J. Duchene, M. Grabisch, D. Hewson, Y. Langeron, and F. Lino, "Evaluation of driver discomfort during long-duration car driving," Applied Ergonomics, Vol. 34, No. 3, 2003, pp. 249-255.

[15] P.A. Fenety, C. Putnam, and J.M. Walker, "In-chair movement: validity, reliability and implications for measuring sitting discomfort," Applied Ergonomics, Vol. 31, No. 4, 2000, pp. 383-393.

[16] G. Kyung, M.A. Nussbaum, and K. Babski-Reeves, "Driver sitting comfort and discomfort (part i): Use of subjective ratings in discriminating car seats and correspondence among ratings," International Journal of Industrial Ergonomics, Vol. 38, No. 5-6, 2008, pp. 516-525.

[17] G. Andreoni, G. C. Santambrogio, M. Rabuffetti, and A. Pedotti, "Method for the analysis of posture and interface pressure of car drivers," Applied Ergonomics, Vol. 33, No. 6, 2002, pp. 511-522.

[18] S. Hermann and H. Bubb, "Development of an Objective Measure to Quantify Automotive Discomfort Over Time," in IEEE International Symposium on Industrial Electronics, 2007. ISIE 2007, 2007, pp. 2824-2830.

[19] G. Kyung and M. A. Nussbaum, "Driver sitting comfort and discomfort (part ii): Relationships with and prediction from interface pressure," International Journal of Industrial Ergonomics, Vol. 38, No. 5-6, 2008, pp. 526-538.

[20] S. Na, S. Lim, H.S. Choi, and M.K. Chung, "Evaluation of driver's discomfort and postural change using dynamic body pressure distribution," International Journal of Industrial Ergonomics, Vol. 35, No. 12, 2005, pp. 10851096.

[21] H.Z. Tan, L.A. Slivovsky, and A. Pentland, "A sensing chair using pressure distribution sensors," Ieee-Asme Transactions on Mechatronics, Vol. 6, No. 3, 2001, pp. 261-268. 